\title{
Introduction to Digital Transformations
}

Technology management has become a powerful tool with the continued increase in emerging technologies. Technology assessment and forecasting is critical for technology managers who seek to reduce the uncertainty created by emerging technologies. With mining tools and the availability of databases, technology mining (TM) has emerged as an effective technology management tool.

Prior research has focused on bibliometrics, patent analysis, and network analysis, as outlined below. This volume tries to integrate these methods to uncover further intelligence.

This book is organized according to the three methods mentioned above. The objective is to demonstrate the application of each tool through multiple cases. We chose cases using the lists published by leading associations and institutes as well as our discussions with colleagues. We recommend the use of multiple approaches to be able to see the whole picture. We believe that the cases chosen represent important technologies enabling a digital transformation of our lives and how we do business.

This book provides important knowledge for the following audiences:

- Industry and government professionals: This book provides multiple benefits for working professionals. First, it demonstrates the tools for mining technological intelligence out of publications and patents. Second, it applies the tools to emerging technologies enabling digital transformation.

- Researchers: This book introduces the researchers in the areas of technology and innovation management. Future research can easily be extended through the case applications in this book. Cases can be analyzed using all three emerging approaches, which can be integrated with traditional approaches such as growth curves or scenarios to provide a deeper analysis for each case.

- Professors: The cases in the book can be used in classes focusing on technology management. The cases would enable discussions about how to identify emerging technologies as well as the leaders in the field.

- Students: Graduate students in programs such as Technology Management, Business Administration, Science and Technology Studies and Engineering Management can use this book as a reference in their studies. 


\section{BIBLIOMETRICS}

Bibliometrics is used to review publications, including journal and conference papers. Reviews of previously published research topics have been used for many years and provide vital intelligence for future research. Prior research includes reviews of many technologies using bibliometrics. Garces et al. (2017) identified leading researchers and institutes in energy-efficient advanced commercial refrigeration using bibliometrics. Behkami and Daim (2012) studied health information technologies using bibliometrics. Marzi et al. (2017) explored the evolution of the literature on product and process innovation through bibliometrics. Finally, Daim and Suntharasaj (2009) integrated bibliometrics into a Bass model to explore technology diffusion. Daim et al. (2006) conducted one of the first studies on the integration of bibliometrics with growth curves. Figures 0.1 and 0.2 provide examples for the topic of fuel cells.

Fuel Cell Bibliometrics - Compendex Database

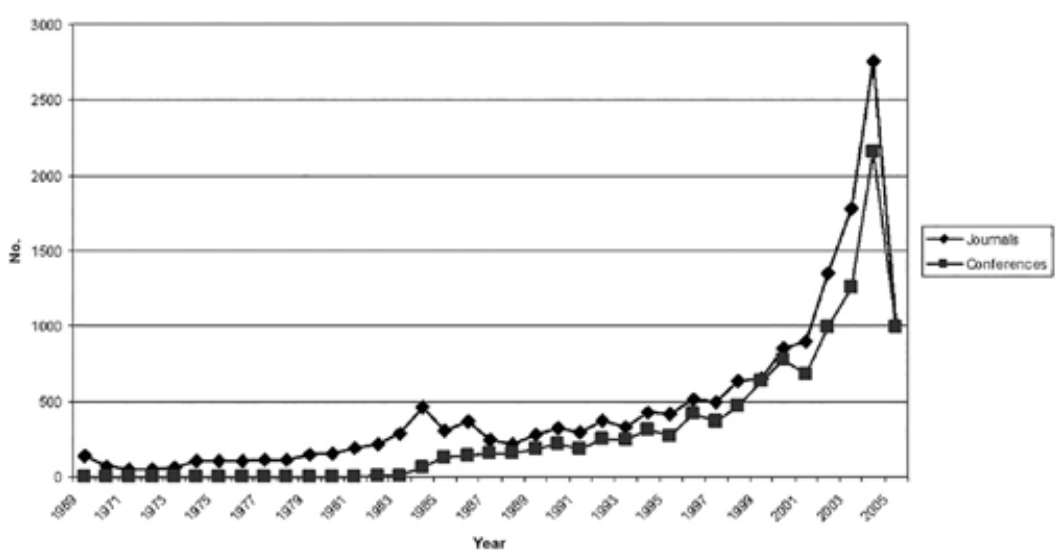

Source: Adopted from Daim et al. (2006).

Figure $0.1 \quad$ Fuel cell bibliometrics - compendex index

There are interesting points to focus on in these types of analyses. Generally, it is misleading to look at the publications from the current year because the total is still cumulating. There are two main kinds of publications. The first are conference publications and the second are journal publications. One must 


\section{Maturity Model}

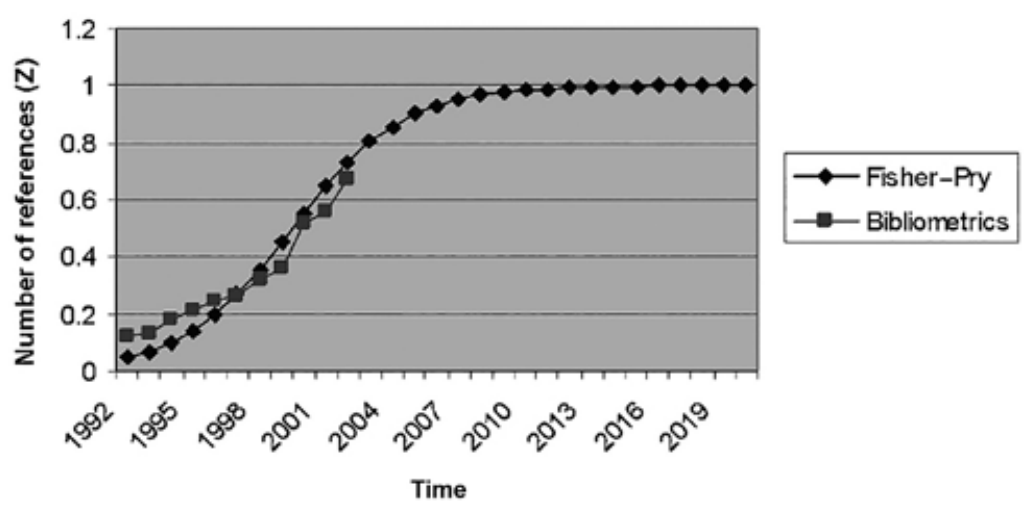

Source: $\quad$ Adopted from Daim et al. (2006).

Figure $0.2 \quad$ Maturity model

be careful in interpreting the results. Journal publications generally take two to three years to appear in indices whereas conference publications appear much faster. Research featured in journal publications may be as dated as six to seven years old. Conferences, on the other hand, publish the most recent research. So, we need to interpret the results considering these timelines.

When we plot publications, we may not see many journal publications in an emerging area whereas conference publications in the same area may be on the rise. Similarly, we may see journal articles increasing in a mature area whereas conference publications may be going down.

Bibliometrics tends to identify activities in the academic world, as industrial actors do not tend to publish papers. Patents are a better indicator of industrial progress.

\section{PATENTS}

While bibliometrics gives us insight into academic progress, patents provide the same intelligence for industrial progress. Prior research has applied this approach to many areas. Fallah et al. (2012) leveraged patent analysis to study the movement of engineers between different companies. Other studies have used patent analysis to explore wind energy (Daim et al., 2012), smart build- 
ings (Madani et al., 2017), televisions (Cho \& Daim, 2016), and electric cars (Gibson et al., 2017).

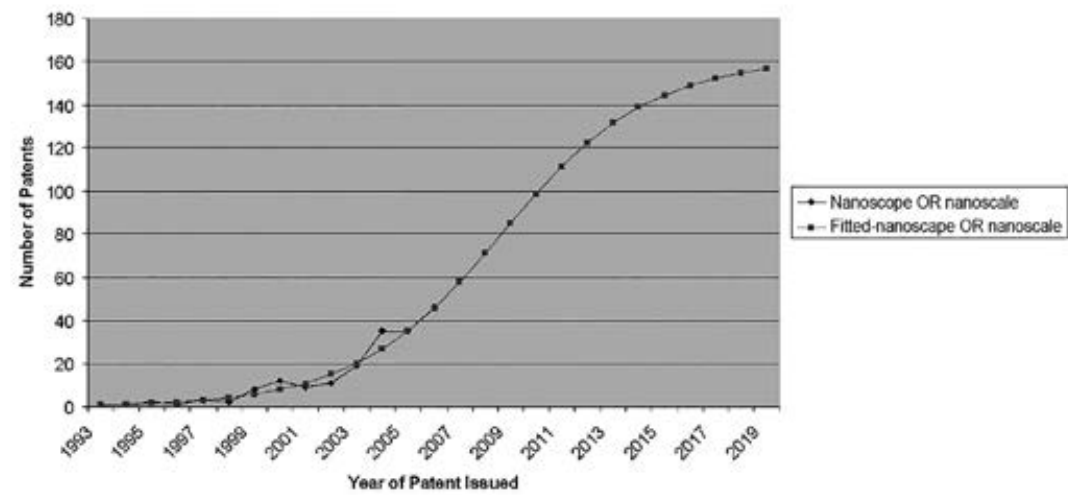

Source: Adopted from Daim et al. (2007).

Figure 0.3 Growth curves for patent data

Figure 0.3 shows how patent data can be used to fit the development to a growth curve, which is also known as an $\mathrm{S}$ curve.

Recent research has advanced the applications of bibliometrics, including leveraging patent data to help with decision making (Gonçalves et al., 2019) and to predict future patent citations (Madani et al., 2018).

\section{NETWORK ANALYSIS}

Network analysis provides depth to intelligence by using either the patent or bibliometrics applications. As demonstrated throughout this book, network analysis can provide metrics for any network and helps us to identify the importance of each node. Recent research has advanced the use of patent analysis alongside network analysis, as with the case of autonomous vehicles studied by Li et al. (2019).

Figures 0.4 and 0.5 provide two metrics of such an analysis applied to technology roadmapping, which is an approach used in technology management.

The data in Figures 0.4 and 0.5 were acquired from the University of Cambridge and the analysis is provided as a demonstration of the approach. 


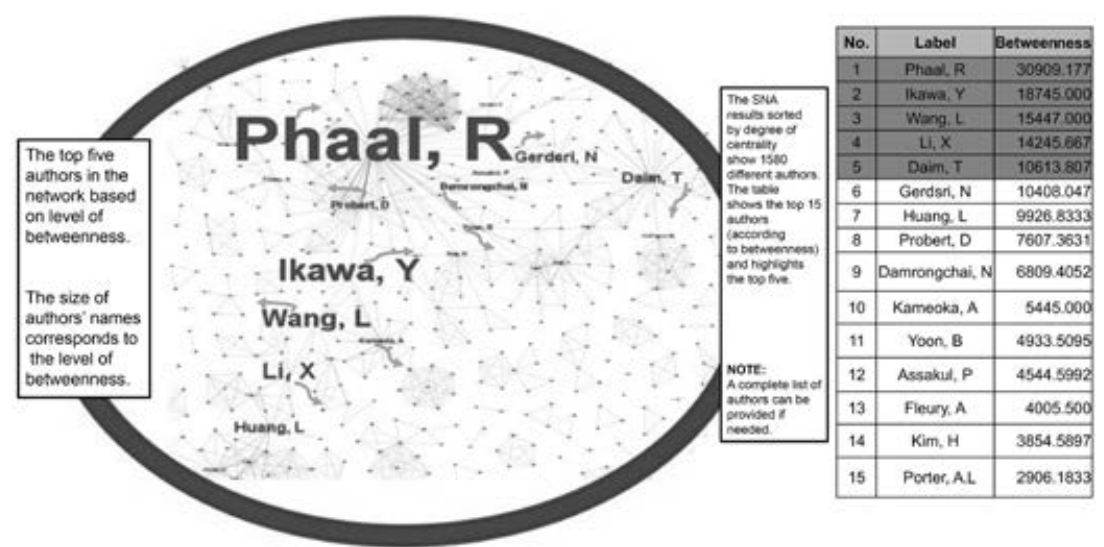

Figure $0.4 \quad$ Authors based on degree centrality

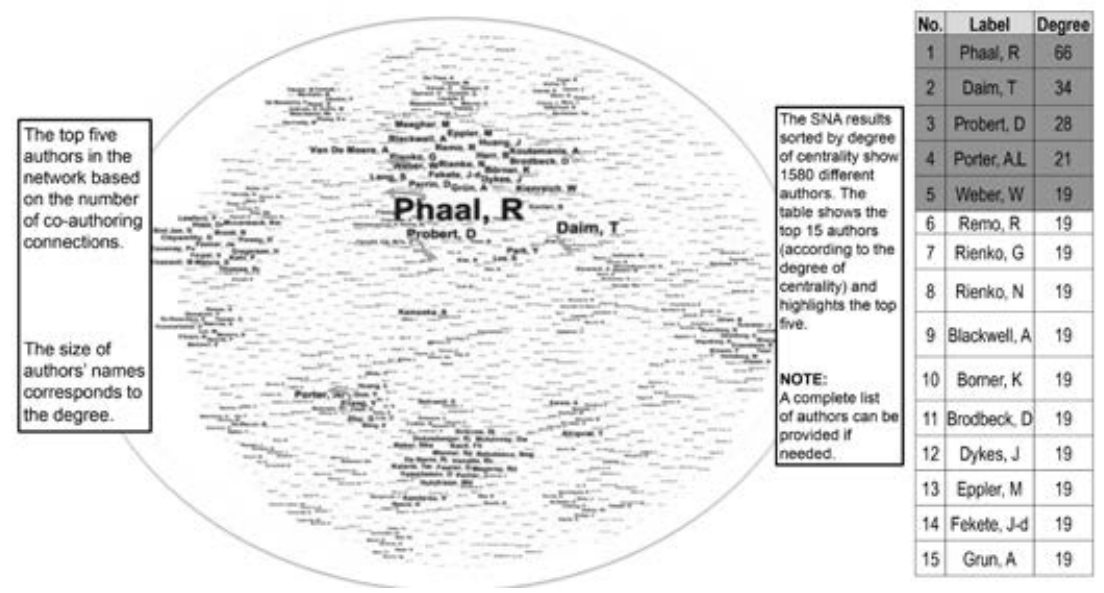

Figure 0.5 Authors based on betweenness centrality

\section{CONCLUSION}

The following table lists the technology cases and corresponding methods used throughout the rest of the book.

As seen in the following table, we tried to focus on emerging technologies and their emerging applications. We were working on this book when the COVID-19 pandemic hit the world. We therefore decided to focus on it in the first chapter. The medical field was considered one of the most important application areas. In addition to the first chapter, four other chapters explore 
Table $0.1 \quad$ Technology case list

\begin{tabular}{l|l}
\multirow{4}{*}{ Bibliometrics } & COVID-19 \\
\cline { 2 - 2 } & Medical AI \\
\cline { 2 - 2 } & Robotic surgery \\
\cline { 2 - 2 } Patent analysis & Transgenic fish \\
\cline { 2 - 2 } & Bioprinting \\
\cline { 2 - 2 } & Medical 3D scanning \\
\cline { 2 - 2 } & Wireless power \\
\cline { 2 - 2 } & Drones in agriculture \\
\hline Network analysis & Automated vehicles \\
\cline { 2 - 2 } & Electric vehicles \\
\cline { 2 - 2 } & Smart homes \\
\cline { 2 - 2 } & Space travel \\
\hline Integrated analysis & Digital twin \\
\cline { 2 - 2 } & Supercomputing \\
\hline
\end{tabular}

applications of emerging technologies in the medical field: artificial intelligence, robotic surgery, bioprinting, and three-dimensional (3D) scanning. We included three chapters focusing on emerging technologies in transportation: space travel and automated and electric vehicles. Finally, the remaining four chapters explore four different fields: transgenic fish, wireless power, drones in agriculture, and smart homes. In addition to these chapters, we have included a chapter on integrated analysis in which two different technologies are examined using all the studied methods and data types. One of the technologies we examine in this section is digital twinning and the other is supercomputing.

When combined, these cases present a major technological transformation of our society. The data collected clearly show that work is underway in many institutes around the world, making us believe that the transformation is on its way.

\section{FUTURE RESEARCH}

The future is already here. Many of the analyses shown in this book can be replicated with other datasets that can be acquired from different databases. For example, Lin et al. (2019) used text mining to explore solar technology. Similarly, Twitter data, litigation data, sponsored research data, and job openings can all be used to identify a perspective on any technology.

We invite all our colleagues to expand and build upon our work and further disclose the progress of digital transformation. 
Tugrul U. Daim and Haydar Yalçın - 9781789908633 Downloaded from PubFactory at 04/26/2023 09:35: 03AM 